\title{
Pharmacological activation of GPR55 improved cognitive impairment, neuroinflammation, oxidative stress and apoptosis induced by lipopolysaccharide in mice
}

\author{
Xin Wang \\ West Anhui Health Vocational college \\ Xiao Tong Xiang \\ Anhui Medical University \\ Jie Hu \\ Anhui Medical University \\ Yu Mei Wu \\ Anhui Medical University \\ YueYue Li \\ Anhui Medical University \\ Shi Yu Jin \\ Anhui Medical University \\ Xian Wu ( $\square$ wuxian2018@126.com ) \\ China Pharmaceutical University
}

\section{Research}

Keywords: Alzheimer's disease, GPR55, Lipopolysaccharide, Cognitive impairment

Posted Date: October 9th, 2020

DOI: https://doi.org/10.21203/rs.3.rs-87517/v1

License: (c) (1) This work is licensed under a Creative Commons Attribution 4.0 International License. Read Full License 


\section{Abstract}

\section{Background}

Neuroinflammation, oxidative stress and apoptosis are implicated in the pathogenesis of Alzheimer's disease (AD). The purpose of the present study was to investigate the neuroprotective effects and possible mechanism of G-protein coupled receptor 55 (GPR55) agonist, 0-1602, on lipopolysaccharide (LPS)-induced cognitive deficits in mice.

\section{Methods}

ICR mice were treated with intracerebroventricular (i.c.v.) injection of LPS. Cognitive tests were performed, including the open field, Morris water maze, novel object recognition, and passive avoidance tests. The expression of GPR55, NF-KB p65, caspase-3, Bax and Bcl-2 were examined in the hippocampus by western blotting. Pro-inflammatory cytokines and microglia were detected by ELISA kit and immunohistochemical analyses, respectively. The malondialdehyde (MDA) level, and superoxide dismutase (SOD) activity were examined by assay kits. Furthermore, TUNEL-staining was used to detect neuronal apoptosis.

\section{Results}

I.c.v. injection of LPS exhibited impaired performance in the behavior tests, which were ameliorated by 01602 treatment(2.0 or $4.0 \mu \mathrm{g} /$ mouse, i.c.v.). Importantly, 0-1602 reversed GPR55 down-regulation, decreased the expression of NF-kB p65, and suppressed the accumulation of pro-inflammatory cytokines and microglia activation, decreased malondialdehyde (MDA) level, and increased superoxide dismutase (SOD) activity. In addition, 0-1602 also significantly decreased Bax and increased Bcl-2 expression as well as decreased caspase-3 activity and TUNEL-positive cells, suppressed neuronal apoptosis in the hippocampus of LPS-treated mice.

\section{Conclusions}

we conclude that 0-1602 may ameliorate LPS-induced cognition deficits via inhibiting neuroinflammation, oxidative stress and apoptosis mediated by NF-kB signaling in mice.

\section{Highlights}

- Activation of GPR55 ameliorates LPS-induced cognitive impairment.

- Activation of GPR55 protects against neuroinflammation, oxidative stress and apoptosis induced by LPS.

\section{Introduction}


Neuroinflammation, oxidative stress and apoptosis are the major processes in the development of Alzheimer's disease (AD)[1-3]. Neurodegenerative diseases including AD are commonly characterized by cognitive dysfunction, which is probably associated with the abnormal activation of neuroinflammation[4]. Oxidative stress is a state in which oxidants produce overwhelming antioxidant defense, which is associated with the onset of $A D[5]$. Increased oxidative stress can cause damage to lipids, DNA, and proteins, leading to decreased neuronal function[6]. Oxidative stress is thought to upregulate the production of amyloid $\beta(A \beta)$ peptide by inducing $\beta$ - and $y$-secretase activity [7].

Lipopolysaccharide (LPS) is an endotoxin isolated from bacteria, which activates the immune system and leads to behavioral and memory impairments, neuroinflammation, and oxidative brain damage $[5,8$, 9]. Previous studies have shown that intracerebroventricular (i.c.v) injection of LPS in mice has been used to establish animal models of memory loss[10,11]. I.c.v administration of LPS in mice produces cognitive impairment by expression of pro-inflammatory cytokines and neuronal death[12]. Considerable evidence has shown that nuclear factor-KB (NF-KB) is a key transcription factor that regulates the expression of proinflammatory cytokines, and the levels of oxidative stress in neurons $[13,14]$. The neuroinflammatory process is characterized by the activation of glial cells such as microglia. Microglia, the brain's resident innate immune cells, is an essential component of neuroinflammatory response [15]. In addition, LPS induces neuroinflammation and oxidative stress-mediated neuronal apoptosis by the activation of caspase $[16,17]$. Caspase-3, has been identified as a key mediator of apoptosis in neuronal cells[18]. Bax, a pro-apoptotic molecule, can induce neuronal cell death while Bcl-2 possesses the opposite effect[19].

G-protein coupled receptor 55 (GPR55) is an orphan G-protein coupled receptor, which is activated by endocannabinoids and lipid transmitters[20,21].The expression of GPR55 in mouse tissue has been declared in the nervous system that includes the hippocampus, though its function remains unknown[22]. GPR55 agonists have been shown to have a neuroprotective effect in Parkinson's disease [23], anxiety[24], and pain perception[25]. It has been reported that activation of GPR55 can strongly protect from ER stress-induced apoptosis in pancreatic $\beta$-cells[26]. 0-1602, a specific GPR55 agonist, can decrease inflammation, improve glycemic control and energy expenditure[27].To date, the effects of GPR55 activation on LPS-induced cognitive deficits, neuroinflammation, oxidative stress and apoptosis have never been examined. In this study, we investigated the neuroprotective effects of 0-1602 on LPSinduced cognitive deficits, neuroinflammation, oxidative stress and apoptosis in mice.

\section{Materials And Methods}

\subsection{Drugs and reagents}

0-1602 was from Intercept Pharmaceuticals. LPS (Escherichia coli serotype 0111:B4) was from SigmaAldrich (St. Louis, USA). Antibodies were from several companies: anti-GPR55 from Abcam (Cambridge, USA); anti-NF-kB p65, anti-caspase-3, anti-Bcl-2, anti-Bax, and anti- $\beta$-actin from Cell Signaling Technology, Inc (Massachusetts, USA). Anti-Histone H3 from Bioworld Technology Co., Ltd (Minneapolis, USA); antiIba1 from Wako Pure Chemical Industries., Ltd (Osaka, Japan). Goat anti-rabbit or anti-mouse IgG-HRP 
(Beyotime, Shanghai, China) was used as the secondary antibody. The nucleoprotein extraction kit was from Sangon Biotech Co., Ltd (Shanghai, China). $\beta$-actin or Histone was used as the internal control for equal loading.

\subsection{Animals}

Male Institute of Cancer Research (ICR) mice, aged 6-week-old and weighing between $25 \mathrm{~g}$ and $28 \mathrm{~g}$ were obtained from the Center of Laboratory Animal of Anhui (Grade II). Mice maintained under standard conditions (12-h-light/dark cycle, 07:00-19:00; ambient temperature, $22 \pm 1^{\circ} \mathrm{C}$; humidity, 40\%) with sufficient supply of food and water. The National Institutes of Health Guide for the Care and Use of Laboratory Animals (NIH Publications No. 80 - 23, revised, 1996) was used for the experiments, and the experimental procedures were approved by the Institutional Animal Care and Use Committee (IACUC) of Anhui Medical University.

\subsection{Experimental groups and animal treatments}

Mice were acclimatized for 7 days and randomized into four groups: vehicle plus vehicle (Veh + Veh), LPS plus vehicle (LPS + Veh), LPS plus 0-1602 (LPS + 0-1602 $2.0 \mu \mathrm{g} / \mathrm{mouse}$ ), and LPS plus 0-1602 (LPS + 0$16024.0 \mu \mathrm{g} /$ mouse). ICV injections of LPS were given using the stereotaxic apparatus according to our previous report [28]. Mice were anesthetized using chloral hydrate $(350 \mathrm{mg} / \mathrm{kg}$, i.p.) and immobilized on a stereotacticframe. The injection volume of $5 \mu$ l of LPS solution or PBS $(1 \mu \mathrm{l} / \mathrm{min})$ was inserted into the mediolateral at a site $1.0 \mathrm{~mm}$ caudal to the bregma, $1.5 \mathrm{~mm}$ from the midline, and $2.0 \mathrm{~mm}$ below the dural surface. The micropipettes were left in place for $5 \mathrm{~min}$ to minimize back flux of liquid. After 3 days, PBS $(5 \mu \mathrm{l})$ with or without 0-1602 was infused into the same site. After 3 days of recovery, some of the mice were subjected to behavioral tests, and the other part of mice was subjected to biological tests.

\subsection{Behavioral analysis}

\subsubsection{Open field test}

Spontaneous locomotor activity was assessed by an open field test prior to the MWM test $[28,29]$. The test was carried out in an open field chamber $(72 \mathrm{~cm} \times 72 \mathrm{~cm} \times 36 \mathrm{~cm})$. Mice were placed in the centre of the open field and allowed a 5-min acclimation period. For each trial, mice were placed in a corner of the device and allowed to explore for $5 \mathrm{~min}$. The total distance, and the time spend in central squares were recorded by an overhead video camera.

\subsubsection{Morris water maze (MWM) test}

Spatial learning and memory function were assessed by MWM test according to our previous studies [30]. It was conducted for 6 days including a 5-days training sessions, and a probe trial on the sixth day. The MWM consisted of a large circular black pool $(120 \mathrm{~cm}$ diameter and $60 \mathrm{~cm}$ height) filled to a depth of $30 \mathrm{~cm}$ with water at $24 \pm 2{ }^{\circ} \mathrm{C}$. The maze was divided into four quadrants, and a clear platform $(9 \mathrm{~cm}$ in diameter) was placed inside. A mounted flag (height, $5 \mathrm{~cm}$ ) was fixed to the platform throughout the visible-platform trial (days 1-2). Each mouse was subjected to four training sessions every day in both 
visible platform and hidden-platform (days 3-5). Mice were released into the water facing the wall of the tank from one of four separate quadrants and were allowed to find the platform within $90 \mathrm{~s}$. If a mouse failed to find the platform in time, the mouse would be guided to the platform. The mouse would be left on the platform to view spatial cues for $30 \mathrm{~s}$, and then returned to the cage. In probe test (day 6 , without flag attached), the mice were allowed to swim in the water tank for $90 \mathrm{~s}$ without the hidden-platform. The trend of the mouse to search for platform was measured by the time spent in the target quadrant, where the platform was previously located. The number of target platform location crossings was recorded by video tracking equipment and processed by a computer equipped with an analysis-management system (ANY-maze video tracking system, stoelting Company, China).

\subsubsection{Novel object recognition (NOR) test}

The recognition memory of mice was evaluated by NOR test as described previously [30, 31]. Mice were placed in the center of the open field chamber $(72 \mathrm{~cm} \times 72 \mathrm{~cm} \times 36 \mathrm{~cm})$. First, mice completed an acquisition trial that consisted of leaving the animals in the apparatus that contained two identical objects. Twenty-four hours later, recognition memory was assessed and a different pair of dissimilar objects (a familiar and a novel one, respectively) were presented. Behavior was recorded by a video camera mounted vertically above the test arena and analyzed using appropriate video-tracking software (Duoyi, Shanghai, China). Object exploration was defined as when the mouse touched or sniffed the object from no more than $2 \mathrm{~cm}$ away. The time spent exploring the two objects were recorded for $5 \mathrm{~min}$. The time spent on the two objects was recorded ( $T$ familiar and $T$ novel). Results were expressed as discrimination index (DI), i.e. ( $T$ novel - $T$ familiar)/ ( $T$ familiar + T novel).

\subsubsection{Passive avoidance test}

The passive avoidance test was carried out in a trough-shaped apparatus consisted of a white illuminated chamber and a dark chamber $(20 \mathrm{~cm} \times 12 \mathrm{~cm} \times 60 \mathrm{~cm}$, respectively). On day 1, mice had a right to explore both chambers for 4 min twice to acclimatize. Next day, the training trial was performed for $5 \mathrm{~min}$ after a 3-min adaption. When mice entered the dark chamber, an electric foot shock (40 V, $2 \mathrm{~mA})$ was delivered. After $24 \mathrm{~h}$, the consolidation trial was performed for $5 \mathrm{~min}$ in the same way as training and the tracking system was started once mice were placed into the light chamber. The step-through latency and error times to enter the dark chamber were recorded [32, 33].

\subsection{Western blot analyses}

The method was performed as described previously [30]. Protein samples $(40 \mu \mathrm{g})$ were added to the band and fractionated by $10 \%$ SDS-PAGE and then transferred to polyvinylidene difluoride membrane(Millipore). Subsequent, membranes were incubated with $5 \%$ non-fat milk containing $0.1 \%$ TBST to block nonspecific binding for $2 \mathrm{~h}$ at room temperature and then incubated at $4{ }^{\circ} \mathrm{C}$ overnight with respective primary antibodies for GPR55 (1:500), caspase-3(1:1000), Bcl-2(1:1000), Bax(1:500), Iba1 (1:1000), and $\beta$-actin(1:2000), respectively, followed by incubation with horseradish peroxidase (HRP)-conjugated Goat anti-Rabbit IgG secondary antibody (1:20000, abcam, USA) at room temperature for $2 \mathrm{~h}$. Nuclear proteins were extracted using nucleoprotein extraction (Sangon Biotech, China).The 
supernatant nuclear protein extract was subjected to western blot for assays of NF-KB p65 (1:1000) and Histone H3 (1:500). The protein bands were visualized using an enhanced chemiluminescence kit (Synthgene Biotech, Nanjing, China). The densities of the bands were quantified by using ImageJ.

\subsection{Enzyme-linked immunosorbent assay (ELISA)}

The homogenate was centrifuged and supernatant was collected carefully and assayed by TNF-a, IL-1 $\beta$ and IL-6 ELISA kit (R\&D Systems, Minneapolis, MN, US) according to the procedures supplied by the manufacturer. Tissue cytokine concentrations are expressed as $\mathrm{pg} / \mathrm{mg}$ of protein [11].

\subsection{Detection of MDA concentration and SOD activity}

We measured the level of MDA and the activity of SOD in the hippocampal tissue with assay kits (Beyotime Biotechnology Institute, Nantong, China). The results are expressed as micromoles of MDA per gram of total protein ( $\mu \mathrm{mol} / \mathrm{g}$ protein) and units per milligram of total protein (U/mg protein), respectively[34].

\subsection{Immunohistochemical (IHC) analyses}

Mouse brain tissue was cut into $30 \mu \mathrm{m}$, then, the sections were immersed in $3 \% \mathrm{H}_{2} \mathrm{O}_{2}$ for $30 \mathrm{~min}$. Sections were blocked with $5 \%$ BSA for $1 \mathrm{~h}$ and then incubated in anti-lba1(1:1000) primary antibody overnight at $4{ }^{\circ} \mathrm{C}$. Next day, sections were incubated with biotinylated mouse anti-rabbit lgG, and incubated with streptavidin-biltin complex (SABC). Diaminobenzidine (DAB) was used as the final chromogen for detection of target proteins. After gradient dehydration, photomicrographs were obtained by a camera and quantified using Image-Pro Plus software. The mean values from 4 sections of each animal were used for statistical analysis.

\subsection{TUNEL staining}

TUNEL staining was performed following the instructions of the in situ cell death detection kit (Roche, Germany). The sections were fixed in 4\% PFA, then washed in PBS ( $\mathrm{pH}$ 7.4). The sections were incubated in permeabilisation solution for $2 \mathrm{~min}$ on ice. TUNEL mixture was added onto brain sections and incubated in a humidified chamber. Sections were incubated for $10 \mathrm{~min}$ in a dark place for DAPI staining. The cells were observed with a fluorescence microscope under a fixed magnification $(\times 200)$ (Leica Microsystems AG, Germany). The number of TUNEL-positive cells in the hippocampal DG region was recorded to evaluate the neuronal apoptosis level (TUNEL-positive cells displayed yellow green fluorescence).

\subsection{Statistical Analysis}

The data are shown as mean \pm standard error of mean (SEM). Group differences were analyzed by a twoway repeated measure ANOVA with "days" as the within-subject factor and "group" as the betweensubject factor in the MWM test. All other data were analyzed by a one-way ANOVA followed by a 
Dunnett's post-hoc analysis for multiple comparisons. All analyses were carried out using SPSS v20.0. $P$ $<0.05$ was considered statistically significant.

\section{Results}

\subsection{0-1602 ameliorates LPS-induced cognitive deficits in mice}

To investigate the effects of 0-1602 on cognitive performance, mice were tested in the open field, NOR, $M W M$, and passive avoidance tests. Firstly, results showed that there was no significant difference among the groups in the total distance traveled and the time spend at the center $(F[3,44]=0.32, P=0.81$; Fig. $1 \mathrm{~A})$, and the time spend at the center $(F[3,44]=0.24, P=0.87$; Fig. $1 \mathrm{~B})$, suggesting that the impaired performance was not due to a decrease in spontaneous locomotor activity.

Next, we assessed the performance of mice in the visible-platform variant (days 1-2) of the MWM test. Mice in each group exhibited similar escape latency (4 trials/mouse/day for 2 days, effect of day, $F[3$, $428]=11.07, P<0.05$; effect of group, $F[3,428]=1.05, P>0.05$; effect of group-by-day interaction, $F[3$, $428]=0.07, P>0.05$; Fig. $1 \mathrm{C}$ ). We then tested the mice in the spatial hidden-platform variant (days $3-5)$, and the data appeared that LPS treatment increased escape latency compared to the control, these were reversed by 0-1602(4 trials/d for $3 \mathrm{~d}$, effect of day, $F[3,620]=13.01, P>0.05$; effect of group, $F[3,620]=$ 2.12, $P>0.05$; effect of group-by-day interaction, $F[3,620]=0.22, P>0.05$; Fig. $1 \mathrm{D})$. In the probe trial (1 trial/mouse for day 6$)$, the data appeared that the mice in the LPS + Veh group displayed a significant decrease in the percentage of time spent in the target quadrant $(F[3,44]=5.47, P<0.01$; Fig. $1 \mathrm{E})$, and the number of platform location crossings $(F[3,44]=6.38, P<0.01$; Fig. $1 \mathrm{~F})$ compared to the control, suggesting memory impairment in the LPS-treated mice. However, mice in the LPS plus 0-1602 group showed significant increases in both indices compared to LPS + Veh group (Fig. 1E and 1F).

To confirm the results observed in the MWM test, we also carried out the NOR and passive avoidance tests. The discrimination index (DI) was calculated as mentioned earlier and shown in Fig. $1 \mathrm{G}$ reveals that the group that received LPS alone showed negative DI values, indicating non-spatial memory impairment where the mice spent more time exploring the familiar object than the novel object $(F[3,44]=4.15, P<$ 0.01 ; Fig. 1G). Notably, treatment with0-1602 showed a significant increase in DI compared with the LPS + Veh group $(P<0.05$; Fig. 1G). The passive avoidance task is a fear-aggravated test used to evaluate nonspatial learning and memory. In the consolidation trial, LPS-induced cognitive deficits were observed as shorter latency and more error times into the dark chamber than those of sham-operated mice $(P<0.05$ or $P<0.01$; Fig. $1 \mathrm{H}$ and I). Compared with LPS-injected group, 0-1602 treatments prolonged the latency, and reversed the increase of error times. Together, these results suggest that LPS induced cognitive impairment, specifically a deficit in memory, which can be ameliorated by 0-1602 treatment. 


\subsection{0-1602 reversed LPS-stimulated GPR55 expression downregulation}

To confirm whether the protective effects of 0-1602 on LPS-induced cognitive deficits were associated with GPR55, the level of GPR55 in the hippocampus was detected by western blot assay. One-way ANOVA revealed that LPS significantly reduced GPR55 expression in the hippocampus, which was reversed by 01602 treatment $(F[3,12]=6.78, P<0.01$; Fig. $2 \mathrm{~A}$ and $\mathrm{B})$. These results suggest that GPR55 might be involved in cognitive deficits induced by LPS in mice.

\subsection{0-1602 inhibits LPS-induced oxidative stress}

MDA is a well-established indicator of lipid peroxidation, and SOD acts as an endogenous scavenger of ROS[34]. Our data indicated that the MDA level was increased $(F[3,12]=6.78, P<0.01$; Fig. $3 A)$ and that SOD activity $(F[3,12]=6.78, P<0.01$; Fig. 3A) was decreased in the LPS group compared to control group. However, treatment with 0-1602 decreased the changes in MDA $(P<0.05)$ and SOD $(P<0.05)$.

\subsection{0-1602 suppresses LPS-activated NF-KB signaling in the hippocampus}

LPS causes memory impairment by the activation of the NF-KB signaling pathway[35]. In this study, nuclear translocation of NF-KB p65 was significantly higher in the hippocampus of LPS-treated mice as compared with the control. However, one-way ANOVA showed that 0-1602 treatment significantly prevented the LPS-induced increased nuclear translocation of NF-KB p65 in the hippocampus $(F[3,12]=$ 13.36, $P<0.01$; Fig. 4 A and B).

\section{5. 0-1602 inhibits the hippocampal proinflammatory cytokines secretion induced by LPS}

Because neuroinflammation is mainly due to the excessive secretion of proinflammatory factors, the levels of TNF- $a$, IL-1 $\beta$, and IL- 6 were detected by ELISA. One-way ANOVA revealed that the levels of TNF- $a$, $\mathrm{IL}-1 \beta$, and IL- 6 were much higher in the LPS group compared with the control group, which was significantly suppressed by 0-1602 treatment $(F[3,12]=13.05, P<0.01$ for TNF-a; Fig. 5A; $F[3,12]=$ 14.46, $P<0.01$ for IL-1 $\beta$; Fig. 5B; $F[3,12]=9.20, P<0.01$ for IL-6; Fig. $5 C)$. These results suggest that 0 1602 inhibited LPS-induced accumulation of proinflammatory cytokines in hippocampus.

\section{6. 0-1602 prevents microglia activation in the hippocampus induced by LPS}

Due to the important role that microglia activation plays in LPS-induced neuroinflammation, we used IHC to investigate microglia activation. The results showed that LPS caused obvious microglia activation in the mouse hippocampal $D G$ region $(F[3,12]=5.92, P<0.01$, Fig. $6 \mathrm{~A}$ and $\mathrm{B})$. This effect was significantly inhibited by $0-1602$ treatment $(P<0.05$ or $P<0.01$; Fig. 6B), suggesting that $0-1602$ could suppress LPSinduced microglia activation in the hippocampus of $D G$ region in mice. 


\section{7. 0-1602 Decreased Lps-induced Neuronal Apoptosis In The Hippocampus}

To determine whether 0-1602 affected neuronal apoptosis, we performed TUNEL assay in the hippocampal DG region. One-way ANOVA revealed that the TUNEL-positive cells numbers of the hippocampal DG region in the LPS + Veh group was significantly higher than the control group $(F[3,12]=$ 9.39, $P<0.01$, Fig. 7B). However, 0-1602 treatment decreased the number of apoptotic cells compared with LPS + Veh group $(P<0.05$ or $P<0.01$, Fig. 7B). The anti-apoptotic effects of $0-1602$ were further investigated using western blot. The results showed that the expression of active caspase-3 was decreased by 0-1602 treatment. The expression of Bcl-2 protein was significantly decreased in the LPStreated mice whereas 0-1602 treatment was able to restore $\mathrm{Bcl}-2$ protein content to that comparable to the Veh + Veh group. The Bax expression was significantly less than that of LPS + Veh group, and the ratio of Bcl-2/Bax was markedly increased after 0-1602 treatment (Caspase-3: $F[3,12]=6.64, P<0.01$; Fig. 7D; Bcl-2/Bax: $F[3,12]=7.94, P<0.01 ; \mathrm{Fig} .7 \mathrm{E})$. These data suggested that $0-1602$ may ameliorate the learning and memory ability by reducing LPS-induced hippocampal neuron apoptosis.

\section{Discussion}

This study showed that 0-1602 prevented LPS induced cognitive deficits, including deficits in spatial learning and memory. The behavioral tests were used as robust and reliable tests that reflect hippocampal dependent learning and memory. In the hidden platform test of MWM, the escape latency of these mice was obviously increased after LPS exposure. For the probe test, LPS-induced mouse model showed significant decrease in the time spent on the target quadrant and the number of target crossings. LPS treatment also caused a significant decline in DI. In the consolidation of the passive avoidance task, LPS-induced cognitive deficits were observed as shorter latency and more error times into the dark chamber than those of sham-operated mice. In addition, LPS treatment in mice led to memory deficit with neuroinflammation, oxidative stress and apoptosis as evidenced by increased nuclear translocation NFKB p65, elevated pro-inflammatory cytokines, activated microglia cells, increased the MDA level, and decreased SOD activity, and increased TUNEL-positive cells, activation of caspase-3, decreased the ratio of Bcl-2/Bax. Importantly, we also found that LPS treatment decreased GPR55 expression in the hippocampus. However, 0-1602 significantly inhibited such adverse cognitive and biochemical changes induced by the i.c.v. injection of LPS. Overall, these suggest that 0-1602 may have potential to protect against LPS-induced cognition impairments in mice.

Increasing evidence indicates that neuroinflammation may play a crucial role in the development of memory deficits[36, 37]. It has been reported that the ROS system is associated with the pathology that results from neuroinflammation[38]. LPS, a stressor produced by gut microbiota, impairs memory and induces inflammatory responses in the hippocampus[39]. I.c.V. administration of LPS in mice produces cognitive impairment by expression of pro-inflammatory cytokines and neuronal death. This method can be effectively used as an animal model for $\operatorname{AD}[29,35,40]$. Expression of several inflammatory genes 
such as inflammatory cytokines, can be regulated by NF-KB activation. Importantly, in this study, 0-1602 treatment prevented improved neuroinflammation by inhibition of inflammatory signaling molecule (NFKB p65) and cytokines (TNF- $\alpha$, IL-1 $\beta$ and IL-6) in the hippocampus of LPS-exposed mice. We speculate that the anti-inflammation effect of 0-1602 might be mediated through NF-KB signaling pathway. Microglia, the brains resident immune cells, are a key element in inflammatory processes and are mediating chronic neuroinflammation and aggravation of AD pathology [41, 42]. Activated microglia are the major source of ROS in the brain[43]. To detect oxidative stress during neuroinflammation induced by LPS and to verify the antioxidative effect of 0-1602, we measured the level of MDA and SOD activity in the hippocampus. In this study, treatment with 0-1602 attenuated the LPS-induced increase in MDA and decrease in the activity of SOD. This result agrees with the evidence that suggests that ROS-induced oxidative stress is one of the most common toxic mechanisms. Furthermore, ROS has been shown to be capable of modulating gene expression. ROS may trigger the generation of cytokines and participate in inflammatory signaling. In addition, NF-KB is central to the acquisition of the pro-inflammatory phenotype, and it is particularly sensitive to ROS[34]. It has been reported that GPR55 expression was observed in microglia [44], and inhibition of NF-KB transcriptional activity in the microglia nucleus suppressed proinflammatory cytokine expression. Thus, our results demonstrated that 0-1602 attenuated inflammatory oxidative stress.

Growing evidence suggests that the potential role of apoptosis in AD has become an area of intense research in recent years[45-47]. LPS-induced neuroinflammation enhances apoptotic neurodegeneration[48]. Early cell changes that occur during apoptosis are mediated by the Bcl-2 family of proteins, including the anti-apoptotic $\mathrm{Bcl}-2$ and pro-apoptotic Bax[16]. In this study, the results are consistent with previous findings. Our results showed that the protein level of the Bcl-2 was decreased; whereas, Bax was increased following LPS administration. Further, treatment with 0-1602 downregulated the expression of Bax and upregulated the expression of Bcl-2 and therefore, mitigated caspase 3 activity and the number of apoptotic cells in LPS-treated mice.

\section{Conclusion}

In summary, our study indicates that 0-1602 displays neuroprotective effects on LPS-induced memory impairment, neuroinflammation, oxidative stress and apoptosis in mice. Therefore, activation of GPR55 has potential therapeutic value for the treatment of neurodegenerative diseases such as AD. However, further investigations are warranted to support this notion and to better understand the exact protective mechanisms of GPR55 in the brain.

\section{Abbreviations}

AD: Alzheimer's disease; GPR55:G-protein coupled receptor 55; LPS:lipopolysaccharide; I.c.v:intracerebroventricular; MDA:malondialdehyde; SOD:superoxide dismutase; A $\beta$ :amyloid $\beta$; NFKB:nuclear factor-KB; ICR:Male Institute of Cancer Research; NOR:novel object recognition; ELISA:enzymelinked immunosorbent assay; IHC:immunohistochemical; 


\section{Declarations}

Acknowledgements

Not applicable.

\section{Funding}

This project was supported by the Doctor Foundation of Anhui Medical University (0601086201), National undergraduate innovation and entrepreneurship training program, and Anhui Medical University School Funding Project.

\section{Availability of data and materials}

The datasets analyzed in the present study are available from the corresponding author on reasonable request.

\section{Authors' contributions}

$\mathrm{XW}$ conceived and designed the study. XTX, JH, YMW, YYL, and SYJ performed the experiments and collected the data. XW analyzed the data, and drafted this article. All authors read and approved the final manuscript.

\section{Ethics approval and consent to participate}

All animal experiments were approved by the Institutional Animal Care and Use Committee at Anhui Medical University.

\section{Consent for publication}

Not applicable.

\section{Competing interests}

The authors declare that they have no competing interests.

\section{References}

1. Janelidze S, Mattsson N, Stomrud E, Lindberg O, Palmqvist S, Zetterberg H, Blennow K, Hansson O. CSF biomarkers of neuroinflammation and cerebrovascular dysfunction in early Alzheimer disease. Neurology. 2018;91:e867-77.

2. Butterfield DA, Halliwell B. Oxidative stress, dysfunctional glucose metabolism and Alzheimer disease. Nat Rev Neurosci. 2019;20:148-60. 
3. Martini F, Rosa SG, Klann IP, Fulco BCW, Carvalho FB, Rahmeier FL, Fernandes MC, Nogueira CW. A multifunctional compound ebselen reverses memory impairment, apoptosis and oxidative stress in a mouse model of sporadic Alzheimer's disease. J Psychiatr Res. 2019;109:107-17.

4. Gao J, Xiong B, Zhang B, Li S, Huang N, Zhan G, Jiang R, Yang L, Wu Y, Miao L, et al. Sulforaphane Alleviates Lipopolysaccharide-induced Spatial Learning and Memory Dysfunction in Mice: The Role of BDNF-mTOR Signaling Pathway. Neuroscience. 2018;388:357-66.

5. Muhammad T, Ikram M, Ullah R, Rehman SU, Kim MO. Hesperetin, a Citrus Flavonoid, Attenuates LPS-Induced Neuroinflammation, Apoptosis and Memory Impairments by Modulating TLR4/NFkappaB Signaling. Nutrients 2019, 11.

6. Tang J, Diao P, Shu X, Li L, Xiong L. Quercetin and Quercitrin Attenuates the Inflammatory Response and Oxidative Stress in LPS-Induced RAW264.7 Cells: In Vitro Assessment and a Theoretical Model. Biomed Res Int. 2019;2019:7039802.

7. Gandhi S, Abramov AY. Mechanism of oxidative stress in neurodegeneration. Oxid Med Cell Longev. 2012;2012:428010.

8. Zarifkar A, Choopani S, Ghasemi R, Naghdi N, Maghsoudi AH, Maghsoudi N, Rastegar K, Moosavi M. Agmatine prevents LPS-induced spatial memory impairment and hippocampal apoptosis. Eur J Pharmacol. 2010;634:84-8.

9. Tripathi A, Paliwal P, Krishnamurthy S. Piracetam Attenuates LPS-Induced Neuroinflammation and Cognitive Impairment in Rats. Cell Mol Neurobiol. 2017;37:1373-86.

10. Ano Y, Ohya R, Kita M, Taniguchi Y, Kondo K. Theaflavins Improve Memory Impairment and Depression-Like Behavior by Regulating Microglial Activation. Molecules 2019, 24.

11. Wang YW, Zhou Q, Zhang X, Qian QQ, Xu JW, Ni PF, Qian YN. Mild endoplasmic reticulum stress ameliorates lipopolysaccharide-induced neuroinflammation and cognitive impairment via regulation of microglial polarization. J Neuroinflammation. 2017;14:233.

12. Bussi C, Peralta Ramos JM, Arroyo DS, Gaviglio EA, Gallea JI, Wang JM, Celej MS, Iribarren P. Autophagy down regulates pro-inflammatory mediators in BV2 microglial cells and rescues both LPS and alpha-synuclein induced neuronal cell death. Sci Rep. 2017;7:43153.

13. Xie C, Li X, Zhu J, Wu J, Geng S, Zhong C. Magnesium isoglycyrrhizinate suppresses LPS-induced inflammation and oxidative stress through inhibiting NF-kappaB and MAPK pathways in RAW264.7 cells. Bioorg Med Chem. 2019;27:516-24.

14. Choi JY, Jang JS, Son DJ, Im HS, Kim JY, Park JE, Choi WR, Han SB, Hong JT. Antarctic Krill Oil Diet Protects against Lipopolysaccharide-Induced Oxidative Stress, Neuroinflammation and Cognitive Impairment. Int J Mol Sci 2017, 18.

15. Fourrier C, Remus-Borel J, Greenhalgh AD, Guichardant M, Bernoud-Hubac N, Lagarde M, Joffre C, Laye S. Docosahexaenoic acid-containing choline phospholipid modulates LPS-induced neuroinflammation in vivo and in microglia in vitro. J Neuroinflammation. 2017;14:170.

16. Goel R, Bhat SA, Hanif K, Nath C, Shukla R. Angiotensin II Receptor Blockers Attenuate Lipopolysaccharide-Induced Memory Impairment by Modulation of NF-kappaB-Mediated 
BDNF/CREB Expression and Apoptosis in Spontaneously Hypertensive Rats. Mol Neurobiol. 2018;55:1725-39.

17. You LH, Yan CZ, Zheng BJ, Ci YZ, Chang SY, Yu P, Gao GF, Li HY, Dong TY, Chang YZ. Astrocyte hepcidin is a key factor in LPS-induced neuronal apoptosis. Cell Death Dis. 2017;8:e2676.

18. D'Amelio M, Cavallucci V, Cecconi F. Neuronal caspase-3 signaling: not only cell death. Cell Death Differ. 2010;17:1104-14.

19. Wang Y, Gu J, Hu L, Kong L, Wang T, Di M, Li C, Gui S. miR-130a alleviates neuronal apoptosis and changes in expression of Bcl-2/Bax and caspase-3 in cerebral infarction rats through PTEN/PI3K/Akt signaling pathway. Exp Ther Med. 2020;19:2119-26.

20. Ryberg E, Larsson N, Sjogren S, Hjorth S, Hermansson NO, Leonova J, Elebring T, Nilsson K, Drmota T, Greasley PJ. The orphan receptor GPR55 is a novel cannabinoid receptor. Br J Pharmacol. 2007;152:1092-101.

21. Oka S, Nakajima K, Yamashita A, Kishimoto S, Sugiura T. Identification of GPR55 as a lysophosphatidylinositol receptor. Biochem Biophys Res Commun. 2007;362:928-34.

22. Sylantyev S, Jensen TP, Ross RA, Rusakov DA. Cannabinoid- and lysophosphatidylinositol-sensitive receptor GPR55 boosts neurotransmitter release at central synapses. Proc Natl Acad Sci U S A. 2013;110:5193-8.

23. Celorrio M, Rojo-Bustamante E, Fernandez-Suarez D, Saez E, Estella-Hermoso de Mendoza A, Muller CE, Ramirez MJ, Oyarzabal J, Franco R, Aymerich MS: GPR55: A therapeutic target for Parkinson's disease? Neuropharmacology 2017, 125:319-332.

24. Rahimi A, Hajizadeh Moghaddam A, Roohbakhsh A. Central administration of GPR55 receptor agonist and antagonist modulates anxiety-related behaviors in rats. Fundam Clin Pharmacol. 2015;29:185-90.

25. Deliu E, Sperow M, Console-Bram L, Carter RL, Tilley DG, Kalamarides DJ, Kirby LG, Brailoiu GC, Brailoiu E, Benamar K, Abood ME. The Lysophosphatidylinositol Receptor GPR55 Modulates Pain Perception in the Periaqueductal Gray. Mol Pharmacol. 2015;88:265-72.

26. Vong CT, Tseng HHL, Kwan YW, Lee SM, Hoi MPM. Novel protective effect of 0-1602 and abnormal cannabidiol, GPR55 agonists, on ER stress-induced apoptosis in pancreatic beta-cells. Biomed Pharmacother. 2019;111:1176-86.

27. Lipina C, Walsh SK, Mitchell SE, Speakman JR, Wainwright CL, Hundal HS. GPR55 deficiency is associated with increased adiposity and impaired insulin signaling in peripheral metabolic tissues. FASEB J. 2019;33:1299-312.

28. Wu X, Lv YG, Du YF, Hu M, Reed MN, Long Y, Suppiramaniam V, Hong H, Tang SS. Inhibitory effect of INT-777 on lipopolysaccharide-induced cognitive impairment, neuroinflammation, apoptosis, and synaptic dysfunction in mice. Prog Neuropsychopharmacol Biol Psychiatry. 2019;88:360-74.

29. Zhao WX, Zhang JH, Cao JB, Wang W, Wang DX, Zhang XY, Yu J, Zhang YY, Zhang YZ, Mi WD. Acetaminophen attenuates lipopolysaccharide-induced cognitive impairment through antioxidant activity. J Neuroinflammation. 2017;14:17. 
30. Wu X, Lv YG, Du YF, Chen F, Reed MN, Hu M, Suppiramaniam V, Tang SS, Hong H. Neuroprotective effects of INT-777 against Abeta1-42-induced cognitive impairment, neuroinflammation, apoptosis, and synaptic dysfunction in mice. Brain Behav Immun. 2018;73:533-45.

31. Fruhauf PK, Ineu RP, Tomazi L, Duarte T, Mello CF, Rubin MA. Spermine reverses lipopolysaccharideinduced memory deficit in mice. J Neuroinflammation. 2015;12:3.

32. Reid SNS, Ryu JK, Kim Y, Jeon BH. GABA-enriched fermented Laminaria japonica improves cognitive impairment and neuroplasticity in scopolamine- and ethanol-induced dementia model mice. Nutr Res Pract. 2018;12:199-207.

33. Wang LS, Tao X, Liu XM, Zhou YF, Zhang MD, Liao YH, Pan RL, Chang Q. Cajaninstilbene Acid Ameliorates Cognitive Impairment Induced by Intrahippocampal Injection of Amyloid-beta1-42 Oligomers. Front Pharmacol. 2019;10:1084.

34. Zhang XY, Xu ZP, Wang W, Cao JB, Fu Q, Zhao WX, Li Y, Huo XL, Zhang LM, Li YF, Mi WD. Vitamin C alleviates LPS-induced cognitive impairment in mice by suppressing neuroinflammation and oxidative stress. Int Immunopharmacol. 2018;65:438-47.

35. Lee HJ, Lim SM, Ko DB, Jeong JJ, Hwang YH, Kim DH. Soyasapogenol B and Genistein Attenuate Lipopolysaccharide-Induced Memory Impairment in Mice by the Modulation of NF-kappaB-Mediated BDNF Expression. J Agric Food Chem. 2017;65:6877-85.

36. Zhao Y, Deng H, Li K, Wang L, Wu Y, Dong X, Wang X, Chen Y, Xu Y. Trans-cinnamaldehyde improves neuroinflammation-mediated NMDA receptor dysfunction and memory deficits through blocking NFkappaB pathway in presenilin1/2 conditional double knockout mice. Brain Behav Immun. 2019;82:45-62.

37. Wu H, Wang X, Gao J, Liang S, Hao Y, Sun C, Xia W, Cao Y, Wu L. Fingolimod (FTY720) attenuates social deficits, learning and memory impairments, neuronal loss and neuroinflammation in the rat model of autism. Life Sci. 2017;173:43-54.

38. Khan MS, Ali T, Kim MW, Jo MH, Jo MG, Badshah H, Kim MO. Anthocyanins protect against LPSinduced oxidative stress-mediated neuroinflammation and neurodegeneration in the adult mouse cortex. Neurochem Int. 2016;100:1-10.

39. Pintado C, Gavilan MP, Gavilan E, Garcia-Cuervo L, Gutierrez A, Vitorica J, Castano A, Rios RM, Ruano D. Lipopolysaccharide-induced neuroinflammation leads to the accumulation of ubiquitinated proteins and increases susceptibility to neurodegeneration induced by proteasome inhibition in rat hippocampus. J Neuroinflammation. 2012;9:87.

40. Liu Y, Zhang Y, Zheng X, Fang T, Yang X, Luo X, Guo A, Newell KA, Huang XF, Yu Y. Galantamine improves cognition, hippocampal inflammation, and synaptic plasticity impairments induced by lipopolysaccharide in mice. J Neuroinflammation. 2018;15:112.

41. Unger MS, Schernthaner P, Marschallinger J, Mrowetz H, Aigner L. Microglia prevent peripheral immune cell invasion and promote an anti-inflammatory environment in the brain of APP-PS1 transgenic mice. J Neuroinflammation. 2018;15:274. 
42. Heppner FL, Ransohoff RM, Becher B. Immune attack: the role of inflammation in Alzheimer disease. Nat Rev Neurosci. 2015;16:358-72.

43. Block ML, Zecca L, Hong JS. Microglia-mediated neurotoxicity: uncovering the molecular mechanisms. Nat Rev Neurosci. 2007;8:57-69.

44. Saliba SW, Jauch H, Gargouri B, Keil A, Hurrle T, Volz N, Mohr F, van der Stelt M, Brase S, Fiebich BL. Anti-neuroinflammatory effects of GPR55 antagonists in LPS-activated primary microglial cells. J Neuroinflammation. 2018;15:322.

45. Zhou TF, Yu JG. Recombinant human erythropoietin attenuates neuronal apoptosis and cognitive defects via JAK2/STAT3 signaling in experimental endotoxemia. J Surg Res. 2013;183:304-12.

46. Shahidi S, AsI SS, Komaki A, Hashemi-Firouzi N. The effect of chronic stimulation of serotonin receptor type 7 on recognition, passive avoidance memory, hippocampal long-term potentiation, and neuronal apoptosis in the amyloid beta protein treated rat. Psychopharmacology (Berl) 2018.

47. Li M, Liu E, Zhou Q, Li S, Wang X, Liu Y, Wang L, Sun D, Ye J, Gao Y, et al: TRPC1 Null Exacerbates Memory Deficit and Apoptosis Induced by Amyloid-beta. J Alzheimers Dis 2018.

48. Lee YJ, Choi DY, Yun YP, Han SB, Oh KW, Hong JT. Epigallocatechin-3-gallate prevents systemic inflammation-induced memory deficiency and amyloidogenesis via its anti-neuroinflammatory properties. J Nutr Biochem. 2013;24:298-310.

\section{Figures}




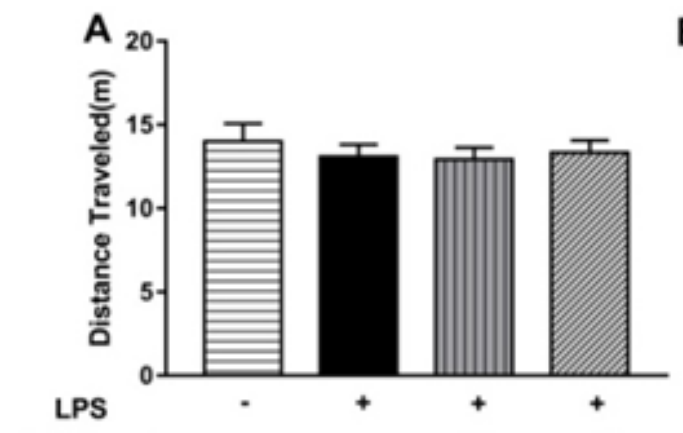

B $0.1602(\mu \mathrm{g})$

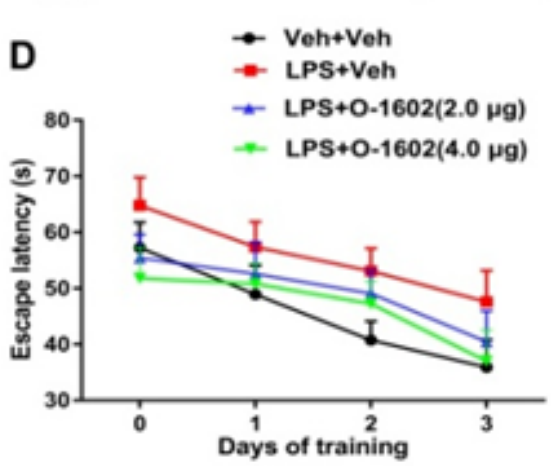

G

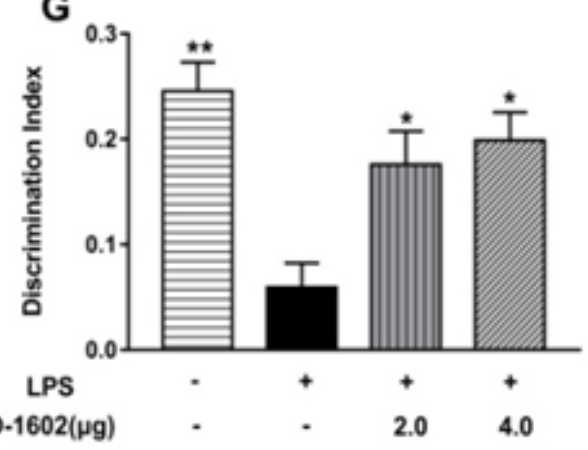

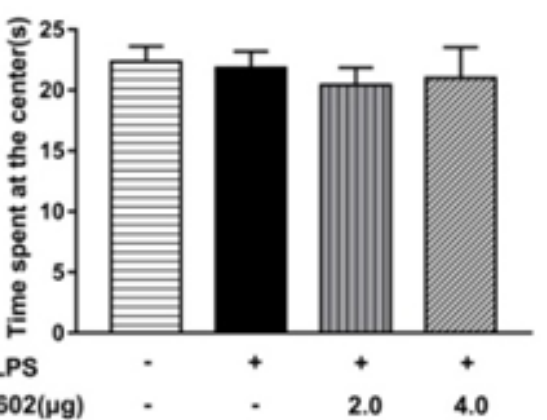

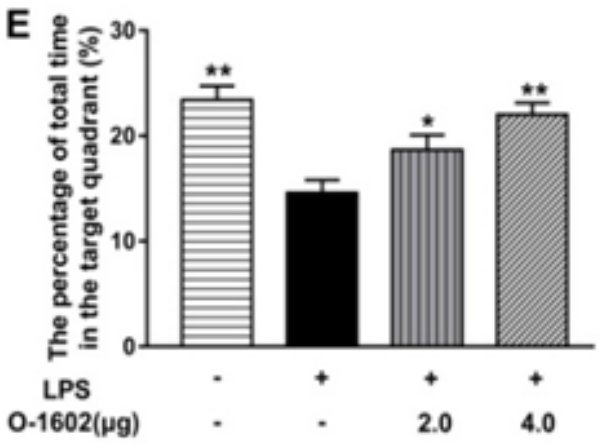

H

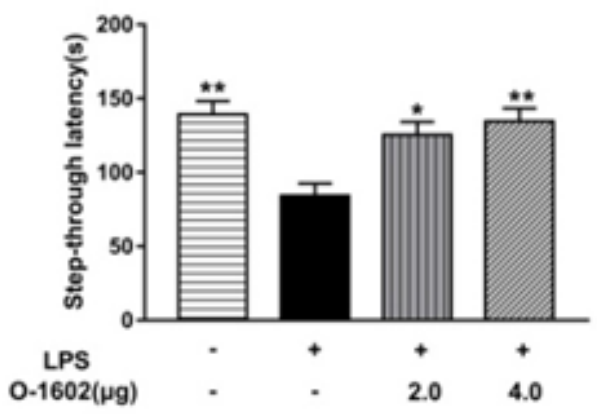

C

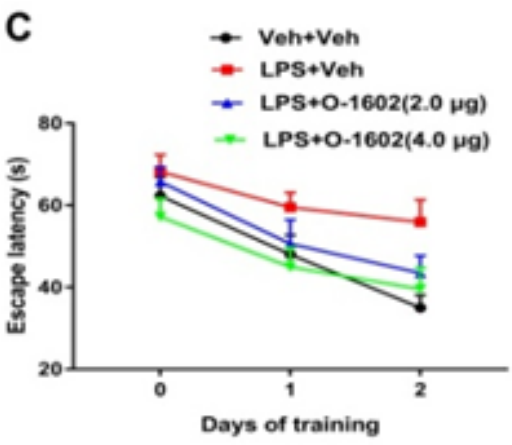

$\mathbf{F}$

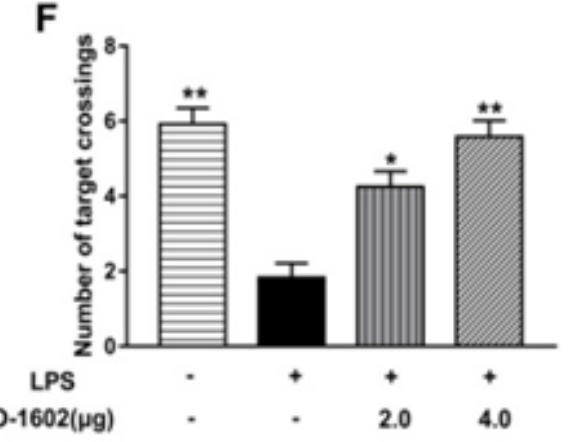

I

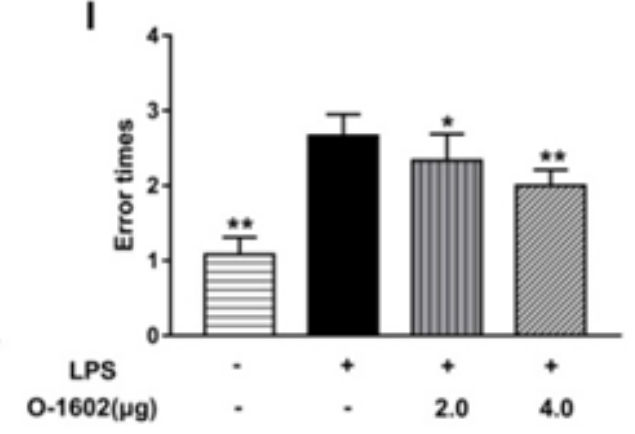

\section{Figure 1}

0-1602 ameliorates LPS-induced cognitive impairment in mice. (A) Distance traveled, (B) time spent in the center zone. (C)The mean escape latency to the visible platform. (D) Latencies to target in training section. (E)Time spent in the target quadrant (\%), and (F) numbers of platform location crossings in the probe trial test. $(\mathrm{G})$ The recognition index. The step-through latency $(\mathrm{H})$ and error times $(\mathrm{I})$ into the dark chamber in the consolidation trial of passive avoidance test. The data are represented as the mean \pm $\mathrm{SEM} ; \mathrm{n}=10-12$. ${ }^{\star} \mathrm{P}<0.05,{ }^{*} \mathrm{P}<0.01$ vs. LPS+Veh group. 


\section{GPR55 \\ (37 kDa)}
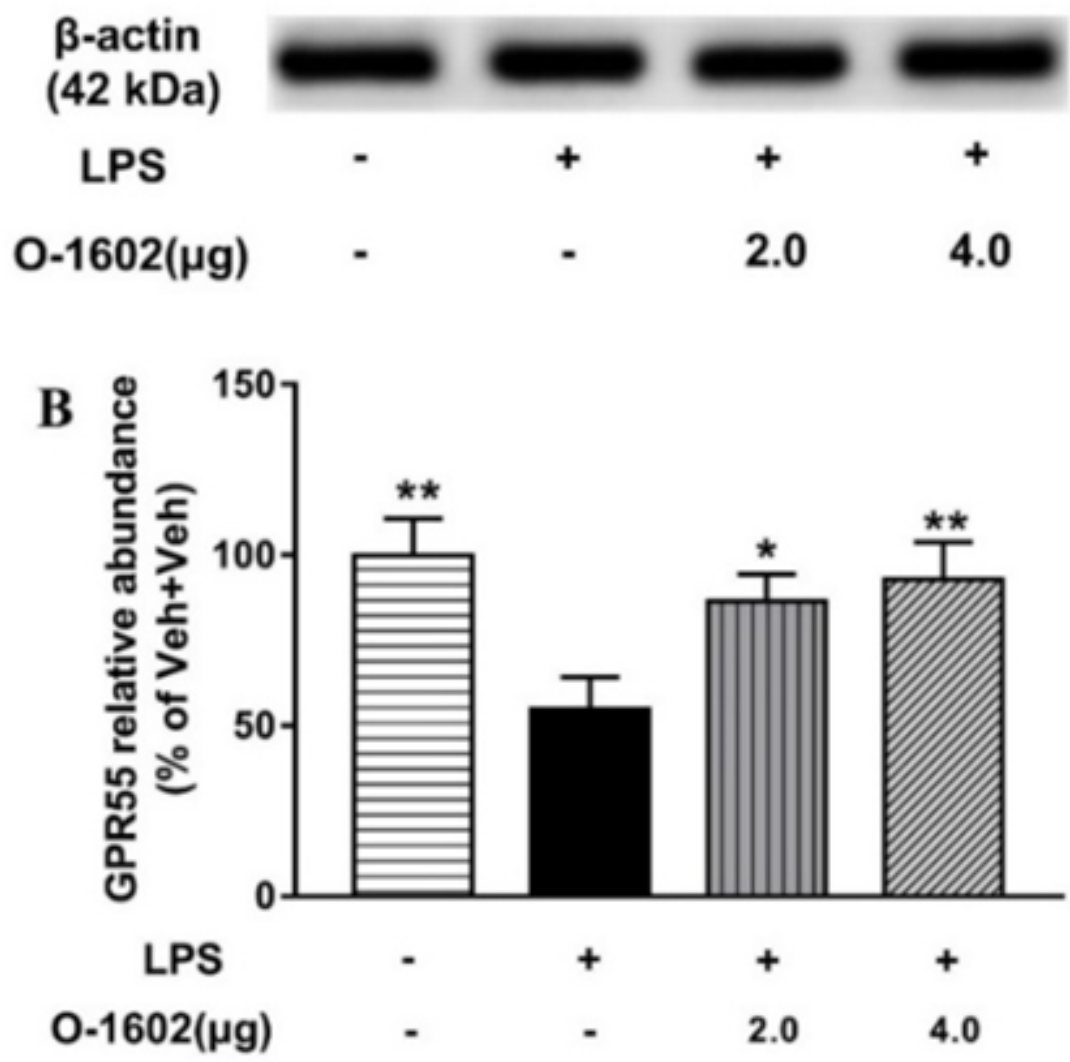

Figure 2

GPR55 expression was down-regulated by LPS exposure and reversed by 0-1602. (A) GPR55 and $\beta$-actin protein bands on the gel and the expression of GPR55 normalized to that of $\beta$-actin as an internal control. (B) Quantification of GPR55 was expressed as the ratio (in percentage) of Veh + Veh group. The data are represented as the mean $\pm S E M ; n=4 .{ }^{*} P<0.05,{ }^{*} P<0.01$ vs. LPS + Veh group.

A

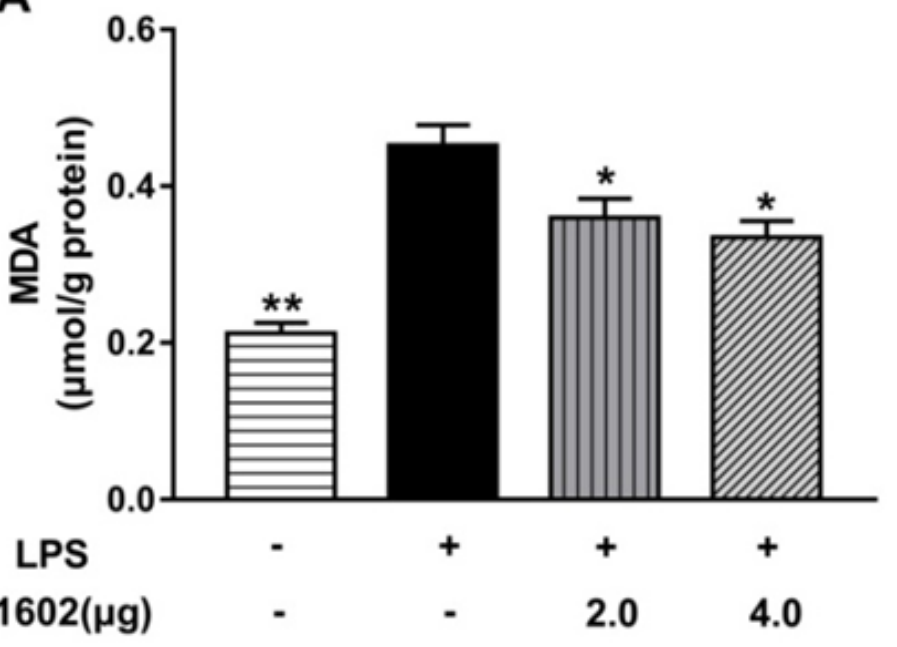

B

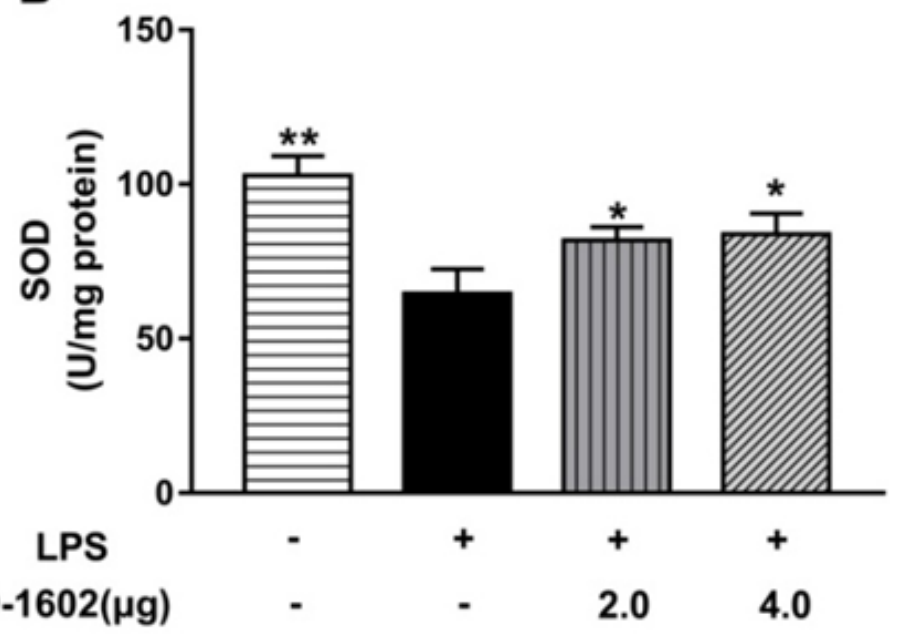


Figure 3

Effects of 0-1602 against LPS-induced oxidative stress in the hippocampal of mouse brains: the level of MDA (A) and the activity of SOD (B). The data are represented as the mean $\pm S E M ; n=4 .{ }^{*} P<0.05$, ${ }^{\star *} P<$ 0.01 vs. LPS+ Veh group.

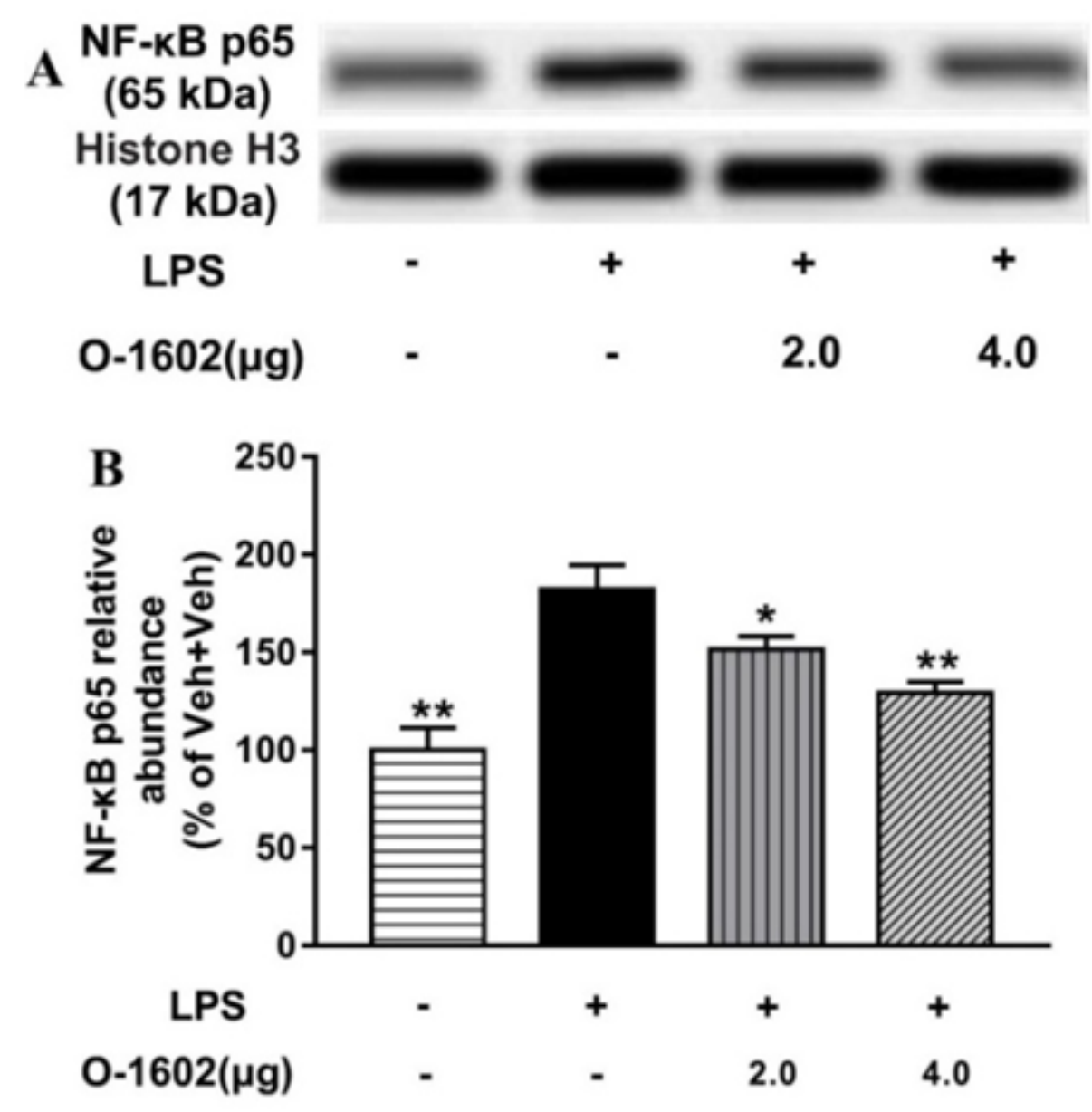

Figure 4

0-1602 suppresses LPS-activated NF-KB signaling in the hippocampus. (A) NF-KB p65 and Histone H3 protein bands on the gel and the expression of NF-KB p65 normalized to that of Histone $\mathrm{H} 3$ as an internal control. (B) Quantification of NF-KB p65 was expressed as the ratio (in percentage) of Veh + Veh group. The data are represented as the mean $\pm S E M ; n=4 .{ }^{\star} P<0.05$, ${ }^{\star \star} P<0.01$ vs. LPS + Veh group. 


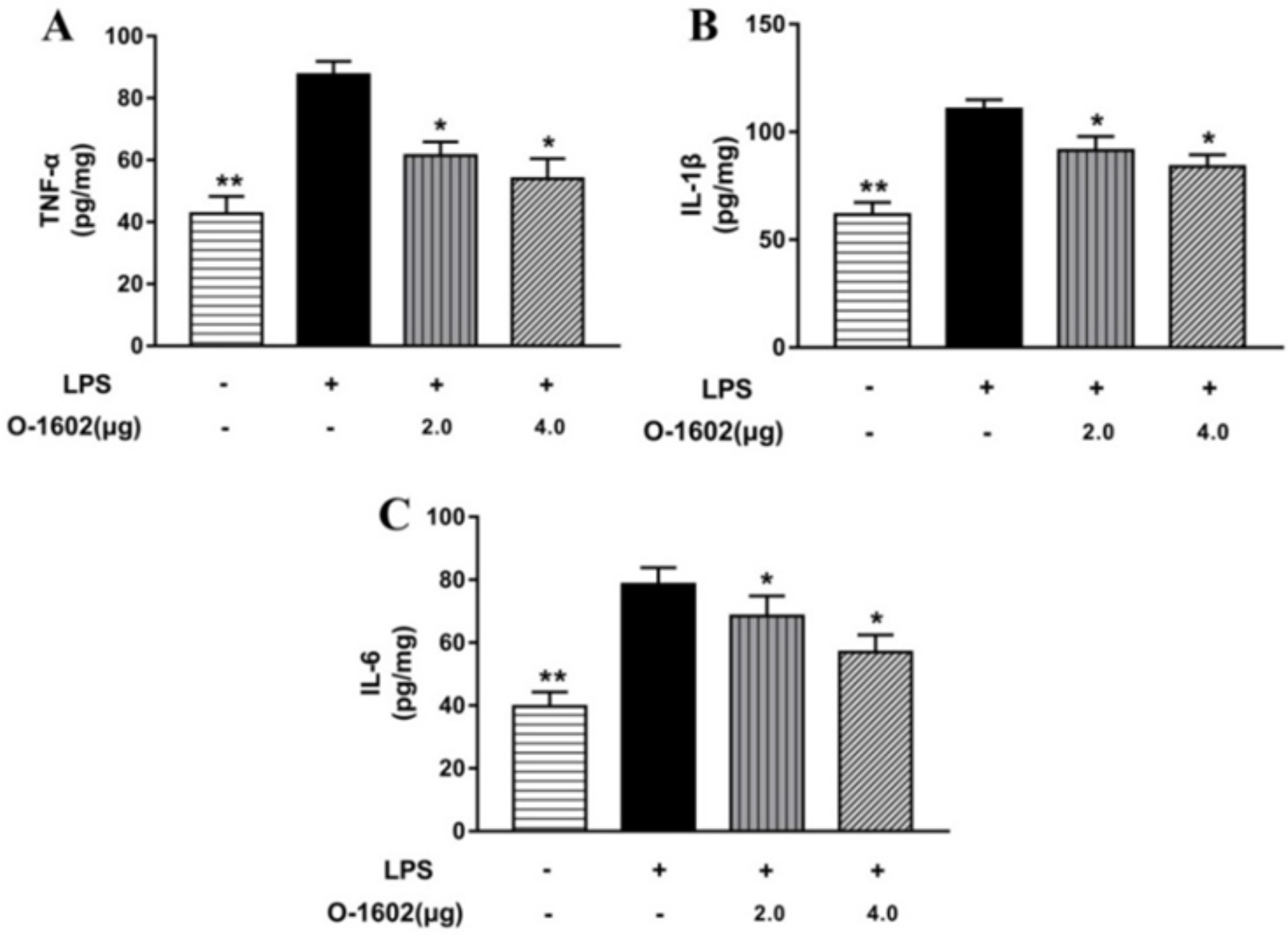

Figure 5

0-1602 decreases the production of proinflammatory cytokines induced by LPS in mice. Quantification of (A)TNF-a, (B) IL-1 $\beta$, and (C)IL-6. The data are represented as the mean $\pm S E M ; n=4 .{ }^{*} P<0.05,{ }^{*} P<0.01$ vs. LPS+ Veh group. 


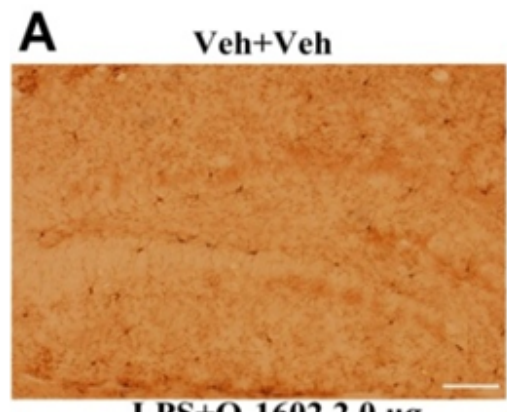

LPS+O-1602 2.0 $\mu \mathrm{g}$
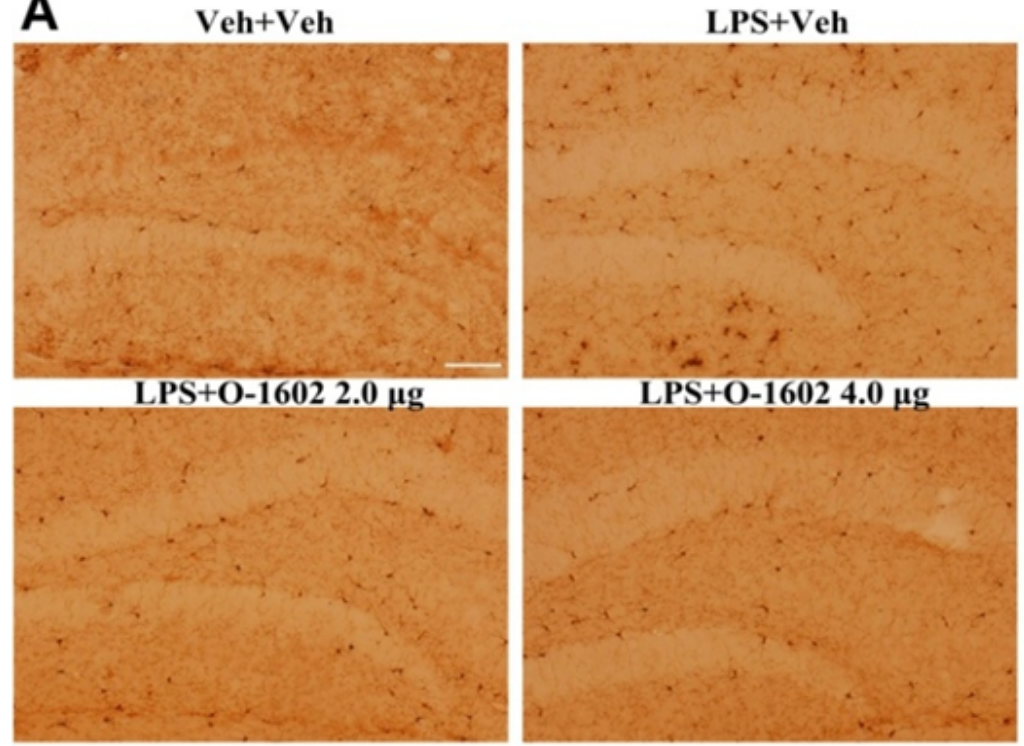

LPS+O-1602 4.0 $\mu \mathrm{g}$

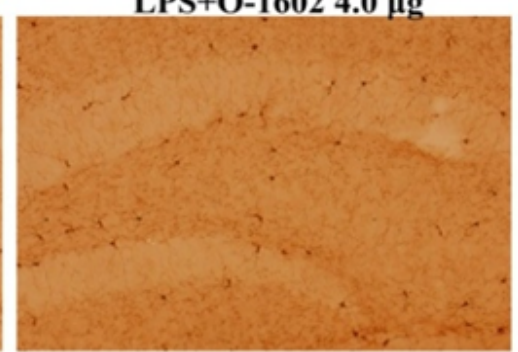

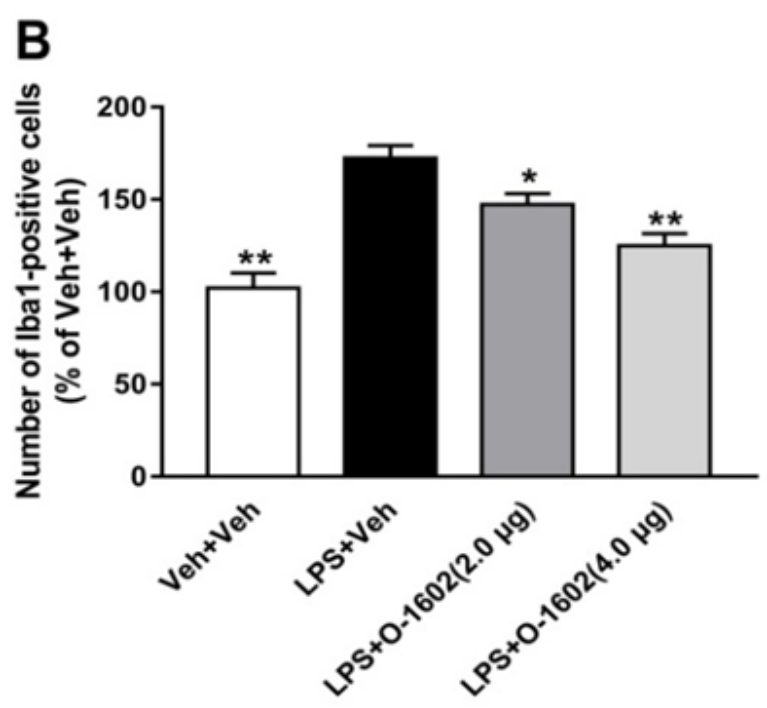

Figure 6

0-1602 attenuates LPS-induced microglia activation in the hippocampus. (A) Representative images of Iba1-labelled activated microglia in the hippocampal DG region. Scale bar represents $100 \mu \mathrm{m}$. (B) The number of Iba1-positive cells. The data are represented as the mean $\pm S E M ; n=4 .{ }^{*} P<0.05$, ${ }^{\star *} P<0.01$ vs. LPS+ Veh group.

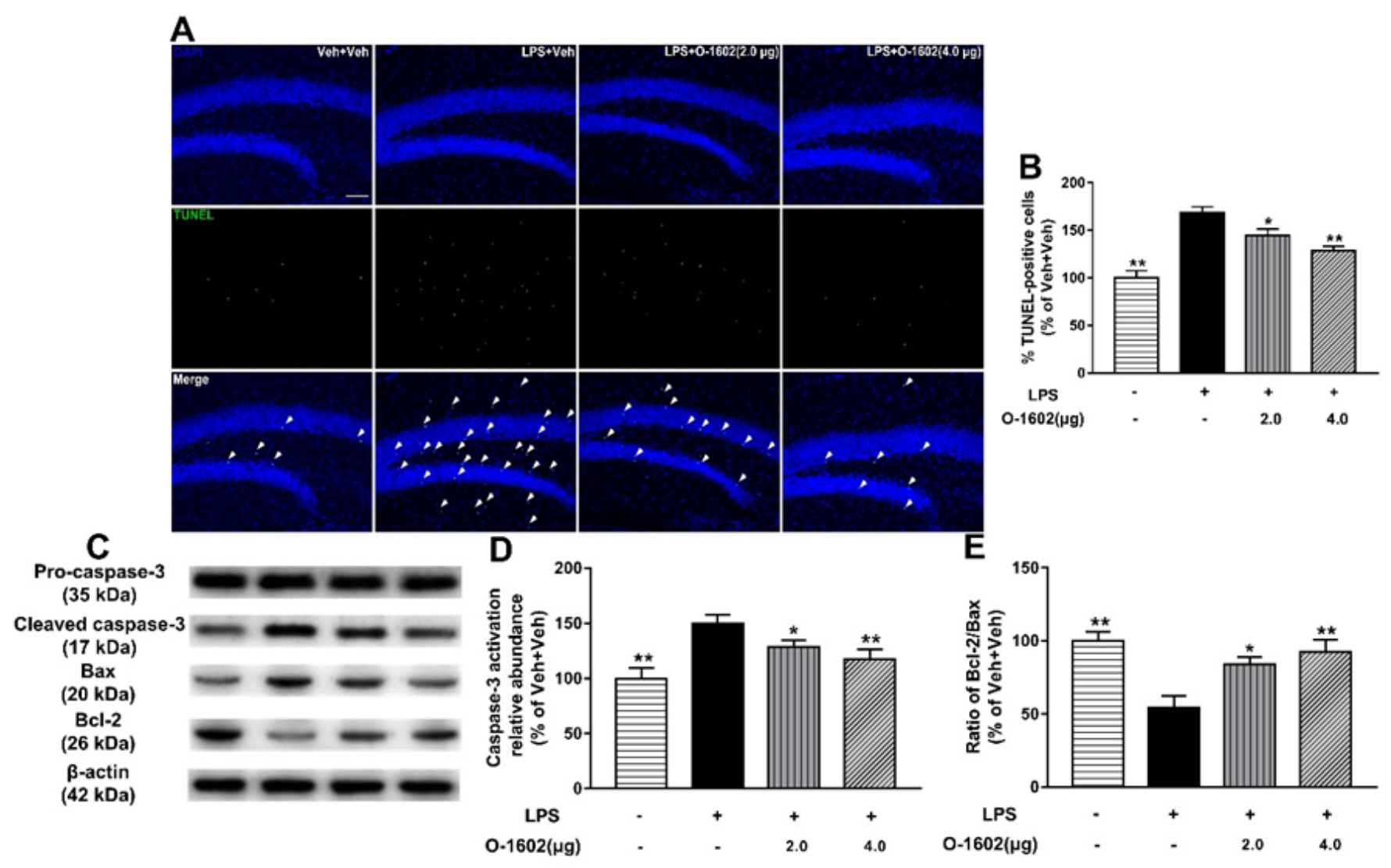

Figure 7 
0-1602 alleviates LPS-induced neuronal apoptosis in the hippocampus. (A) Representative images of TUNEL-positive cells in the hippocampal DG region. Scale bar $=100 \mu \mathrm{m}$. (B) The apoptotic bodies were expressed as a percentage of the total number of cells, and were expressed as the ratio (in percentage) of Veh + Veh group. (C) Pro-caspase-3, cleaved caspase-3, Bax and Bcl-2 and $\beta$-actin protein bands on the gel and the expression of pro-caspase-3, cleaved caspase-3, Bax and Bcl-2 normalized to that of $\beta$-actin as an internal control. (D) Caspase-3 activation was expressed as the ratio of caspase- 3 fragment to procaspase-3. (E) Ratio of Bcl-2/Bax was expressed as the ratio (in percentage) of Veh+ Veh group. The data are represented as the mean $\pm S E M ; n=4 .{ }^{*} P<0.05$, ${ }^{\star *} P<0.01$ vs. LPS + Veh group. 